\title{
Entre Casandra y Clío: A propósito del pasado, el presente y el futuro de la filosofía de la historia
}

\author{
Óscar Moro Abadía
}

Ecole des Hautes Etudes en Sciences Sociales, París

\begin{abstract}
RESUMEN. Tomando como referencia la publicación de la segunda edición del libro de Concha Roldán Entre Casandra y Clío, el presente artículo examina algunas cuestiones relacionadas con el pasado, el presente y el futuro de la filosofía de la historia. En primer lugar, se analiza la crisis actual que atraviesa la disciplina tratando de determinar cuál puede ser el sentido de la filosofía de la historia en el comienzo del siglo XXI. En segundo lugar, se pasa revista al debate a propósito del carácter científico del conocimiento histórico y se examinan sus implicaciones en la actualidad. En tercer lugar, se intenta determinar la función ética que puede desempeñar la filosofía de la historia.
\end{abstract}

Palabras clave: Filosofía de la historia, Filosofía especulativa de la historia, filosofía crítica de la historia, Historia y ciencia, Ética.

\section{Introducción}

La publicación a finales de 2005 de la segunda edición del libro de Concha Roldán Entre Casandra y Clío constituye una excelente ocasión para debatir a propósito del pasado, el presente y el futuro de la filosofía de la historia. Al fin y a cabo, tal y como la autora señala en la introducción, el texto es una invitación a pensar la filosofía de la historia a la luz
ABSTRACT. Taking the publication of the second edition of Concha Roldan's Entre Casandra y Clio as reference, this article analyses the philosophy of history from the perspectives of the past, the present and the future. First, the paper examines the current disciplinary crisis in an effort to define the philosophy of history at the beginning of the twenty-first century. Second, the article considers the historical dimension of the debated scientific nature of historical knowledge and includes the current implications of this controversy. Third, the essay explores the ways in which the philosophy of history can play an ethical function.

Key words: Philosophy of history, speculative philosophy of history, critical philosophy of history, History and Science, Ethics.

de su crisis actual: "Los autores y los problemas tratados no son fruto del azar, sino que responden a una clara intención, la de mostrar en qué puede consistir la filosofía de la historia hoy, a través de una historia en la que se ponen de manifiesto los excesos cometidos por esta disciplina, excesos que la llevaron a granjearse el rechazo que sufre en la actualidad"1. En otras palabras, ya desde buen comienzo el libro se coloca bajo el 
signo de esa tríada consustancial a la propia filosofía de la historia: el pasadopresente- futuro.

En primer lugar, Entre Casandra y Clío constituye una de las mejores historias de la filosofía de la historia escritas en lengua castellana. Frente a otros trabajos que repasan el pasado de la disciplina en un tono más ensayístico ${ }^{2}$ o que plantean otras alternativas ${ }^{3}$, el texto de Roldán permite obtener una visión de conjunto de la historia de la disciplina. Desde San Agustín hasta Collingwood, pasando por Voltaire, Vico o Hegel, el lector encuentra en estas páginas una exposición clara y precisa del pensamiento de aquellos autores que contribuyeron a forjar esa particular forma de reflexión sobre la historia que fue la Geschichtsphilosophie ${ }^{4}$. En este sentido, Roldán hace un buen trabajo de síntesis al definir el concepto "filosofía de la historia", limitarlo históricamente y establecer cuáles han sido sus condiciones de posibilidad. En segundo lugar, la reflexión de Roldán está orientada por una actualidad que, desde Hegel, se ha convertido en una de las tareas principales de la filosofía ${ }^{5}$. Se trata, en definitiva, de examinar el pasado a la luz del presente, tomando como referencia la crisis de la disciplina para determinar si podemos seguir hablando de "filosofía de la historia" y, si es así, en qué sentido debemos hacerlo. En tercer lugar, Entre Casandra y Clío es una invitación a pensar sobre el porvenir de la disciplina. La crisis actual nos coloca frente a un futuro incierto donde la filosofía de la historia sólo podrá sobrevivir a condición de aceptar que "se ha roto en mil pedazos el espejo de lo absoluto"6 y que es necesario buscar nuevos parámetros para pensar filosóficamente la historia. Como la propia autora señala, aunque las concepciones tradicionales tienen que ser descartadas, lo cierto es que algún tipo de reflexión filosófica sobre la historia es, y seguirá siendo, pertinente y necesaria.

Para plantear este pequeño ensayo a propósito del pasado, el presente y el futuro de la filosofía de la historia, me gustaría retomar tres de las principales preguntas a las que el libro de Roldán pretende responder. A través de ellas espero establecer un diálogo con la autora que me permita, en la aurora del siglo XXI, tomar el pulso a la Philosophie der Geschichte. En primer lugar, la cuestión a propósito de la crisis de la filosofía de la historia: ¿Por qué la filosofía de la historia ha llegado a ser una disciplina tan discutida? ¿Es legítimo seguir utilizando dicha denominación? Es suficiente con echar un vistazo al libro de Roldán para constatar que, hasta fecha reciente, el pulso entre filósofos e historiadores se resolvió a favor de los primeros: "En su afán por explicar el conjunto ha perdido de vista los aspectos singulares e individuales de la disciplina que comenzó siendo su objeto de estudio. La filosofía de la historia [...] estaba concebida como materia de especulación metafísica, de ahí que sea conocida entre nosotros como filosofía especulativa de la historia"7. Esa concepción especulativa de la disciplina ${ }^{8}$ (que Walsh denomina filosofía especulativa de la historia y Danto filosofía substantiva $)^{9}$ se encuentra desde hace tiempo ${ }^{10}$ seriamente discutida: "Ha acabado la concepción de una historia entendida como un proceso único, evolutivo, coherente hacia una meta"11. Del mismo modo, el énfasis en la búsqueda de explicaciones científicas y de leyes generales que caracterizó a la filosofía crítica de la historia también ha sido puesto en entredicho. Ante esta situación, la actualidad nos obliga a hacer frente a la crisis de las filosofías tradicionales para tratar de construir una nueva reflexión sobre la historia que bien pudiera adoptar la forma de una filosofía 
crítica, entendida como "renuncia a esperar el sentido último de la evolución" ${ }^{12}$. Mi primer objetivo será, por tanto, determinar qué sentido puede tener una filosofía de la historia en los inicios del siglo XXI.

Un segundo problema que el libro plantea es la pregunta a propósito del carácter científico de la historia: ¿Es la historia una ciencia? Si es así, ¿debe adoptar el modelo de las ciencias naturales o de las ciencias sociales? Estas son preguntas a las que historiadores y filósofos se han enfrentado desde hace casi dos siglos. Para unos, como Leopold von Ranke $^{13}$ o Louis Bourdeau ${ }^{14}$, la historia tenía que renunciar a toda especulación metafísica y convertirse en una ciencia natural que mostrase los hechos "tal y como sucedieron" (Wie es eigentlich gewesen). Para otros, como Wilhem Dilthey ${ }^{15}$, la historia tenía que construirse a partir de la metodología de las ciencias del espíritu y no de las ciencias naturales. Esta polémica, que se antoja intrínseca al propio saber histórico, se ha reactivado recientemente gracias a la controversia en torno al "giro lingüístico". La cuestión será, por tanto, retomar el pasado de esta discusión para examinar los ecos de la misma en el presente. Al fin y al cabo, toda reflexión filosófica sobre la historia que se pretenda crítica tiene, necesariamente, que hacer frente al problema de la cientificidad y a otras cuestiones derivadas (el problema de la verdad, de la objetividad, de la existencia de leyes generales, etc.).

La tercera cuestión que me gustaría examinar es la relación entre historia y ética: ¿Puede seguir Casandra profetizando al oído de Clío? ¿Se le puede exigir a la filosofía de la historia algún tipo de compromiso ético hoy que parecemos instalados en el escepticismo, ya sea moderno o postmoderno? Roldán considera que la filosofía de la historia, toda vez liberada de su condición de gran relato omnicomprensivo, está en condiciones de desempeñar una función ética: "El filósofo de la historia ya no puede dedicarse a realizar terroríficas o esperanzadoras predicciones de futuro, pero tampoco debe renunciar a realizar valoraciones estimativas acerca del mismo: no puede anunciar lo que será, pero sí proponer cómo debiera ser o, en todo caso, cómo no debiera ser jamás"16. En este contexto, mi objetivo será plantear algunas preguntas a propósito de esa dimensión ética de la disciplina. Quizás, como analizaré más adelante, la filosofía de la historia pueda convertirse en una reflexión capaz de orientar la acción en el presente.

Las cuestiones que acabo de mencionar (la crisis de la filosofía de la historia, la naturaleza científica de la historia y la relación entre filosofía de la historia y ética) me servirán como piedras de toque para tomar el pulso de ese paciente aparentemente moribundo que es la filosofía de la historia.

\section{La crisis de la filosofía de la historia}

No creo que me equivoque cuando afirmo que el primer objetivo de Entre Casandra y Clío es responder a una serie de cuestiones a propósito del presente de la filosofía de la historia: “¿Acaso [...] la filosofía de la historia es algo que sólo pertenece al pasado? ¿Podemos seguir hablando de filosofía de la historia? Si es así, ¿en qué sentido?"17. Sin embargo, como la autora nos recuerda, para hacer frente a dicha actualidad es necesario mirar hacia atrás, hacer recuento e intentar dar respuesta a una pregunta siempre pertinente: ¿Cómo la filosofía de la historia ha llegado a ser lo que es? En su búsqueda de respuestas, el libro de Roldán parte de una actitud crítica hacia el pasado disciplinar: no se trata de restituir 
la gloria perdida a los padres fundadores sino de poner de manifiesto los errores cometidos en el pasado.

Roldán considera que pueden distinguirse cuatro grandes fases en la historia de la filosofía de la historia: una larga prehistoria que se prolonga desde la Grecia Clásica hasta el Renacimiento; una aurora que anuncia las ideas fundamentales sobre las que se construirá la disciplina; una tercera fase que corresponde al apogeo de la filosofía especulativa de la historia y, por último, un período donde se desarrolla una filosofía de la historia que se pretende crítica no sólo por poner en tela de juicio los planteamientos anteriores sino también por concentrarse en el análisis de una serie de problemas concretos.

Durante las dos primeras fases de esta cronología (i.e. desde la Antigüedad hasta el siglo XVI) no puede hablarse de una filosofía de la historia sensu stricto. En los orígenes del pensamiento occidental, la historia y la filosofía siguieron caminos paralelos que impidieron que una filosofía de la historia pudiera constituirse. De hecho, ambas disciplinas remitían a dos tipos de saber contradictorios. Por un lado, la historia (historia rerum gestarum) no podía ser objeto de especulación filosófica porque se limitaba a la simple narración de los hechos por parte de un testigo de los acontecimientos. Por otro lado, la duración no era susceptible de convertirse en objeto filosófico: el filósofo era aquel capaz de adquirir conocimientos científicos, es decir conocimientos inmutables no sujetos al cambiante devenir. Habría que preguntarse si, como señala Cassirer citando a Burckhardt, toda la filosofía de la historia posterior no quedó lastrada por esta contradicción inicial: "La filosofía de la historia, dice Burckhardt, es un centauro, una contradicción en los términos porque la historia coordina y por esta razón es no filosófica, mientras que la filosofía subordina y por esta razón es antihistórica"18.

Esta situación de incompatibilidad se mantuvo hasta la obra de San Agustín, creador de una primera filosofía cristiana de la historia. En mi opinión, Roldán acierta al considerar que De Civitate Dei constituye la primera interpretación sobre el sentido de la historia universal, pero que todavía no puede considerarse filosofía (puesto que no es una visión racional de la historia). De hecho, sólo en el Renacimiento comenzó a desarrollarse un tipo de pensamiento sobre el pasado que dará lugar, durante la Ilustración, a la filosofía de la historia sensu stricto. El nacimiento de esta reflexión se explica principalmente por "la emancipación de una razón filosófica respecto de una tradición teológica que la venía encorsetando y que, sin el proceso de secularización nunca habría alcanzado su mayoría de edad"19. Las obras de Leibniz, Voltaire, Vico y Herder son consideradas el precedente inmediato de una filosofía de la historia que alcanzará su apogeo a finales del siglo XVIII y principios del XIX gracias a Kant y Hegel, y que se prolongará durante el siglo XIX de la mano de Comte y de Marx.

La filosofía especulativa de la historia es un tipo de reflexión que puede definirse a partir de los siguientes rasgos: 1) tiene por pretensión explicar el conjunto de la historia ${ }^{20}, 2$ ) define la historia como un proceso universal $^{21}, 3$ ) equipara el proceso histórico a la realización de la razón, 4) busca determinar las leyes que demuestren que la historia no es una secuencia arbitraria de hechos, 5) pretende predecir los acontecimientos futuros, 6) está organizada en torno a las ideas de evolución y de progreso. Esta visión de la historia (que, grosso modo, es común a Kant, Hegel, Comte y Marx) está construida sobre una distinción entre los 
hechos y la Historia: "Los "hechos" son sólo datos, indicios, síntomas en los que aparece la realidad histórica. Ésta no es ninguno de ellos, por lo mismo que es fuente de todos. Más aún: que "hechos" acontezcan depende, en parte, del azar. Las heridas de César pudieron no ser mortales. Sin embargo, la significación histórica del atentado hubiera sido la misma"22. En definitiva, se trataba de un tipo de reflexión construida sobre el olvido de la historia o, si se prefiere, de espaldas a los hechos históricos. Tanto la idea de una historia universal de Kant, como la ley de los tres estadios de Comte o la dialéctica (ya sea en su versión hegeliana o marxista) eran reconstrucciones ideales a priori sobre el devenir histórico.

Esta concepción entró en crisis a finales del siglo XIX con el paulatino rechazo de las concepciones metafísicas hegelianas. A partir de ese momento, la disciplina experimentó un cambio fundamental. Para ilustrarlo, quisiera examinar brevemente el libro de Oswald Spengler Der Untergang des Abendlandes (La decadencia de Occidente) que bien pudiera considerarse, si se me permite el juego de palabras, der Untergang der Geschichtsphilosophie. Esta obra constituyó el último intento de construir una filosofía especulativa de la historia que, en la fecha de aparición del primer volumen (junio de 1918), ya no era posible. Spengler, cuyo objetivo fundamental era predecir la historia ${ }^{23}$, comienza planteando la división entre los hechos históricos y la realidad histórica: “¿Hay una lógica de la historia? ¿Hay más allá de los hechos singulares, que son contingentes e imprevisibles, una estructura de la humanidad histórica, por decirlo así, metafísica, que sea en lo esencial independiente de las manifestaciones político-espirituales tan patentes y de todos conocidas?'24. El autor alemán descubre esa "estructura de la humanidad histórica" en la "cultura", verdadera protagonista de la historia. Spengler distingue nueve culturas, cada una de las cuales "imprime a su materia, que es el hombre, su forma propia, cada una tiene su propia idea, sus propias pasiones, su propia vida, su querer, su sentir, su morir propios" 25 . Oponiéndose a la imagen tradicional de la historia como una línea recta, Spengler ve múltiples culturas que se suceden llenando el tiempo histórico. Utilizando la metáfora orgánica, considera que dichas culturas son seres vivos que nacen, maduran, envejecen y mueren. El objetivo del libro es analizar el último estadio de una de esas culturas: Occidente. En definitiva, lo que la obra de Spengler pone de manifiesto es que el a priori del progreso que había organizado la filosofía especulativa de la historia ya no era válido en 1918: "el porvenir del Occidente no consiste en una marcha hacia delante sin término, en la dirección de nuestros ideales presentes y con espacios fantásticos de tiempo, sino que es un fenómeno normal de la historia, limitado en su forma y duración"26. La idea de un plan, de un propósito, de una meta que guía el destino de la humanidad (noción intrínseca a la filosofía especulativa) había sido definitivamente descartada: "La humanidad no tiene un fin, una idea, un plan, como no tiene fin ni plan la especie de las mariposas o de las orquídeas"27.

El rechazo de la filosofía idealista llevó al interés por una filosofía de la historia que no fuera interpretada en términos ni místicos ni metafísicos. Es lo que Walsh llama la filosofía crítica de la historia que se interesa por el estudio de cuatro conjuntos de problemas: a) la historia y otras formas de conocimiento, b) verdad y hecho en historia, c) objetividad histórica y d) la explicación en historia $^{28}$. Se trata, como Roldán señala, “de 
un planteamiento fundamentalmente epistemológico, que atañe a la posibilidad de conocimiento de la historia"29. Es por esta razón que Ernst Cassirer habla de un "enfoque epistemológico de la historia" 30 que ya no estaría interesado en la historia como objeto, sino en el modo de conocimiento de dicho objeto. Se pretendía, por tanto, determinar el método de conocimiento o, si se prefiere, el carácter específico del conocimiento histórico. Esta filosofía crítica de la historia (que nació en el siglo XIX) se prolongó durante el siglo XX metamorfoseada en una filosofía analítica principalmente interesada en determinar si la historia es o no una ciencia. Roldán sitúa la crítica popperiana del historicismo y las propuestas de Hempel, Dray y Von Wright en este contexto (2005, pp. 143-155).

Lo significativo es que algo de la pretensión omnicomprensiva y normativa de la denostada filosofía especulativa sobrevivió en la filosofía crítica: "La filosofía crítica de la historia, que todavía en alguna de sus más recientes versiones ha tendido a autoconcebirse como una reflexión de orden superior sobre la metodología historiográfica, acaparando para si y sometiéndolo a una tan férrea cuanto injustificada voluntad normativa ese impreciso territorio conocido como la teoría de la historia" 31 . En definitiva, el intento de conocer científicamente la historia (i.e. la búsqueda de leyes generales) escondía la pretensión de predecir el futuro y, sobre todo, negaba la impredicibilidad de la historia. Sin embargo, también el énfasis positivista en la explicación se saldó con un fiasco del que da fe el fracaso del Covering Law Model de Hempel.

Llegados a este punto, me gustaría retomar la pregunta que dio origen a esta reflexión: si tanto la filosofía especulativa como la filosofía crítica de la historia parecen haber fracasado, ¿tiene sentido seguir hablando de una "filosofía de la historia"? Dado que se trata de una cuestión fundamental, me gustaría explorar las dos respuestas posibles. En primer lugar, parece evidente que hoy no podemos pensar la historia en términos siquiera similares a los de la filosofía especulativa. La creencia en el progreso, el establecimiento de leyes generales o la posibilidad de predecir el futuro son ideales gnoseológicos que pertenecen a un tiempo pasado. Del mismo modo, "la expectativa objetivista debiera considerarse definitivamente descartada" 32 , y con ella se han esfumado parte de las ilusiones, en el doble sentido del término, de la filosofía crítica. En definitiva, las formas tradicionales de pensar filosóficamente la historia no han podido adaptarse a los nuevos tiempos. Esto ha provocado que, no sin razón, algunos autores hayan rechazado el término "filosofía de la historia" por considerarlo demasiado ligado a la visión tradicional de la Geschichtsphilosophie.

Sin embargo, hay quienes creemos que "sigue habiendo algo a lo que denominados filosofía de la historia, sólo que ha cambiado de signo [...] continúa pensándose sobre y a partir de la historia, como si los filósofos hubieran experimentado un giro desde lo perenne a lo perentorio"33. En otras palabras, si consideramos la filosofía de la historia como un intento de pensar sobre y a partir de la historia, entonces estaremos de acuerdo en que dicha reflexión no sólo es pertinente sino que es necesaria (llámese "filosofía de la historia", llámese "metodología histórica" como prefiere decir Manuel Cruz). Dado que los viejos modelos ya no sirven como referencia, la cuestión estribaría en llenar de contenido dicha reflexión. Para ello, hay que partir de un hecho fundamental: estamos en un momento en el que se produce más pensamiento sobre la historia que nunca. El 
problema es, como ha afirmado Javier Muguerza, que los tiempos no parecen estar para sistemas ${ }^{34}$ y que esa ingente reflexión se nos presenta bajo formas tan diversas que apenas podemos adivinar su sentido. Por esta razón, pienso que una de las funciones de la filosofía de la historia debería ser, precisamente, explicar esa multiplicidad. Se trataría, en definitiva, de que la filosofía nos ayudase a comprender qué tipo de historia escribimos, por qué y en qué medida esa reflexión está orientada por la actualidad. Ese trabajo de síntesis podría permitirnos comprender el vínculo privilegiado que la historia establece con el presente ${ }^{35} \mathrm{y}$, de este modo, ayudarnos a entender nuestro hoy.

En segundo lugar, la filosofía de la historia debe ayudar a pensar los conceptos, las ideas y las categorías del tiempo que nos ha tocado vivir. Desde luego, ya no es posible imaginar la historia como la realización del progreso o de la razón. Tampoco parece conveniente salir a la búsqueda de pautas y leyes generales que expliquen el pasado y predigan el futuro. Ahora bien, como Roldán señala, eso no significa que la reflexión filosófica sobre la historia haya muerto, sino que ha cambiado de signo. Nuestro tiempo es el de la multiplicidad, el escepticismo y la diversificación. En él han surgido nuevos objetos de pensamiento que hace apenas dos siglos eran despreciados. La literatura, cada día más abundante, a propósito de la contingencia, el acontecimiento, la narración, la identidad o la memoria da fe de la existencia de una reflexión fértil sobre la historia. Sería un error, desde mi punto de vista, negarle a dicha reflexión la categoría de pensamiento por el mero hecho de que no adopte una forma sistemática. Nuestra tarea debería ser, por tanto, construir una filosofía acorde con nuestro tiempo, aunque sepamos que no es definitiva ${ }^{36}$.

\section{Historia y ciencia}

El debate a propósito del status científico de la historia se remonta al siglo XIX y ha adoptado dos formas diferentes: las discusiones entre los filósofos en torno a la cuestión de la historia como ciencia (¿Es la historia una ciencia del mismo rango que las naturales? ¿Es posible la predicción en historia?, etc.) y los debates entre los historiadores que, más ligados a la práctica de la historia, han intentado determinar las posibilidades de construir un relato fidedigno del pasado. Dado que el texto de Roldán examina en detalle la primera de estas dos formas, he pensado que lo mejor sería completar el cuadro trazado por la autora recogiendo las principales discusiones entre los historiadores. De este modo, quizá estemos en condiciones de responder a una cuestión que Iggers planteaba del siguiente modo: "¿Qué queda hoy de la fe de la Ilustración en la posibilidad de ocuparse racionalmente, esto es, científicamente, de la historia y que queda del cometido emancipador de la ciencia ahora, cuando la posmodernidad ha cuestionado radicalmente no sólo la coherencia de la historia sino también la del pensamiento humano?"37.

Durante el siglo XIX, la historia se constituyó como una disciplina universitaria que, como Janus, presentaba dos caras: la científica y la política. En efecto, desde muy pronto la actividad de los historiadores se inscribió en un campo de fuerza resultado de la tensión entre estos dos polos. Por un lado, aspiraban a producir un relato verdadero sobre el pasado. El "noble sueño" de Ranke tenía su correlato en el deseo de "permanecer independientes de toda opinión política y religiosa $[\ldots]$ sin proponerse otro objetivo y otro fin que el que puede obtenerse de la verdad" 38 . Por otro lado, la institucionalización de la historia "no puede 
separarse de la experiencia nacional que dicha historia pretende presentar como natural y evidente" 39 . El caso francés es un ejemplo paradigmático de la liaison entre el proceso de legitimación del Estado moderno y el nacimiento de la profesión de historiador. Así, la burguesía que pasó a detentar el poder a partir del siglo XIX comprendió que la nueva sociedad, "para creer en ella misma, tenía necesidad de no haber nacido ayer"40. Era necesario justificar el nuevo estado revolucionario, colocándolo "bajo el peso del pasado de Francia"41. En este sentido, la historia se convirtió en un instrumento de legitimación fundamental que tenía como uno de sus principales objetivos "extraer de nuestras viejas crónicas relatos capaces de emocionar la fibra popular" 42 .

Estos dos procesos (la búsqueda de la verdad y la utilización política del pasado) instauraron una paradoja en el corazón mismo de la empresa histórica moderna: por un lado, la historia se constituyó como un saber que tenía como función principal legitimar el nuevo orden político y, por otro, los historiadores aspiraban a restituir la verdad de los tiempos pasados por encima de intereses políticos concretos. Durante más de un siglo, esta contradicción marcó la orientación de los debates teóricos en la disciplina, en los que cuestiones como la objetividad y la responsabilidad se convirtieron en problemas fundamentales. Sin embargo, durante mucho tiempo, los historiadores tuvieron la capacidad no de superar la mencionada paradoja, pero si al menos de soportar el peso de la contradicción. De hecho, pienso que, hasta finales del siglo $\mathrm{XX}$, los historiadores fueron capaces de encontrar un equilibrio entre la búsqueda de la verdad y las implicaciones políticas de su actividad. En primer lugar, aprovecharon el diálogo con otras ciencias sociales para construir una historia capaz de ofrecer un relato verídico del pasado. A pesar de que la cuestión de la historia como ciencia fue objeto de discusión permanente (el texto de Roldán resume perfectamente los principales debates al respecto), lo cierto es que nadie ponía en duda que el historiador fuera capaz de ofrecer un retrato fidedigno del pasado. En segundo lugar, los historiadores guardaron una cierta autonomía con respecto al poder político. Constituidos en comunidad universitaria, fueron capaces de consolidar un campo relativamente independiente de las demandas políticas. En este sentido, como cualquier otro campo científico, el de los historiadores puede ser considerado "un microcosmos autónomo en el interior del macrocosmos social. Autónomo, según la etimología, quiere decir que tiene su propia ley, su propio nomos, que tiene en sí mismo el principio y la regla de su funcionamiento" 43 . En definitiva, el equilibrio ${ }^{44}$ que hizo posible el desarrollo de la historia durante el siglo XX estuvo basado en dos principios fundamentales: (a) el acuerdo a propósito del carácter verdadero y riguroso del conocimiento histórico y (b) la consolidación de una cierta autonomía de los historiadores con respecto a los poderes políticos.

Sin embargo, está situación ha cambiado durante los últimos años. De hecho, creo que lo que los especialistas llaman la "crisis de la historia" es un proceso justamente relacionado con la quiebra de los dos principios que hacían posible el mencionado equilibrio. En primer lugar, desde los años setenta ciertas corrientes de pensamiento han puesto en cuestión la posibilidad misma de construir un relato verídico sobre el pasado. Ya no se trata de saber si la historia es una ciencia del mismo rango que las naturales, sino de determinar si el discurso histórico es una forma de retórica 
próxima a la literatura. En segundo lugar, la creencia en la autonomía del historiador ha desaparecido. Así, escuchamos hablar constantemente de la utilización política de la historia, de los usos del pasado o de los abusos de la memoria. La idea de que la historia "siempre tiene una función política" 45 se ha generalizado. Dado que estos dos procesos parecen haber puesto en jaque la posibilidad del conocimiento racional del pasado, me gustaría detenerme brevemente en ellos.

En primer lugar, desde la publicación del libro de Hayden White Metahistory. The Historical Imagination in Nineteenth-Century Europe 46 y del artículo de Lawrence Stone The Revival of Narrative: Reflections on a New Old History ${ }^{47}$, un número cada vez mayor de historiadores parecen convencidos de que la historia tiene más que ver con la literatura que con la ciencia. Así, el último desafío a la historia científica proviene del "giro linguístico" (linguistic turn) ${ }^{48}$, una forma de textismo popularizada por el propio Hayden White. Según él, la historia es una estructura lingüística (verbal structure) que no difiere esencialmente de la literatura. Por consiguiente, "los historiadores tienen que tomar las narrativas históricas por lo que manifiestamente son: ficciones verbales cuyos contenidos tienen más en común con sus homólogos literarios que con los de las ciencias"49. Tomando como referencia algunas ideas de la filosofía del lenguaje de Wittgenstein ${ }^{50}$, los defensores del giro lingüístico consideran que el historiador no hace referencia al pasado, sino que construye la realidad histórica.

En segundo lugar, durante los últimos años se ha insistido mucho en el uso político de la historia. Aunque los historiadores han tenido conciencia de dicha utilización desde hace más de un siglo, lo cierto es que fue a partir de la década de los setenta cuando se generalizó la idea de que la única función de la historia ha sido (es y será) legitimar el poder. Esta "pérdida de la inocencia" no es un fenómeno exclusivo de la historia: forma parte de una toma de conciencia más general a propósito de las implicaciones políticas de las ciencias sociales. Desde la aparición de la obra de autores como Said o Foucault, las ciencias sociales han retenido la idea de que "el poder produce saber [...] que poder y saber se implican directamente el uno al otro; que no existe relación de poder sin constitución correlativa de un campo de saber, ni de saber que no suponga y no constituya al mismo tiempo unas relaciones de poder" 51 .

Tanto el giro lingüístico como la generalización en la creencia del carácter político del discurso histórico nos obligan a replantearnos una serie de cuestiones fundamentales: ¿Pueden los historiadores superar el desafío postmoderno ${ }^{52}$ ¿Pueden volver a alcanzar el equilibrio perdido? ¿Es todavía posible una historia científica? La filosofía de la historia se antoja un espacio privilegiado desde el que responder a estas cuestiones. En primer lugar, puede aportar un diagnóstico bien informado a propósito de problemas tales como la objetividad, la verdad o la explicación (cuestiones que son objeto de debate desde hace un siglo). En definitiva, puede introducir la reflexividad necesaria para comprender la deriva de la discusión. En segundo lugar, la filosofía de la historia pueda ayudar a encontrar un tercer camino que nos permita escapar del callejón en el que relativistas y positivistas nos han metido. Se trataría de aceptar tanto la imposibilidad de la objetividad como la determinación socio-lingüística del conocimiento ${ }^{53}$ sin caer en el relativismo absoluto. Sólo si resolvemos esa ecuación estaremos en condiciones de avanzar. 


\section{Filosofía de la historia y ética}

La tercera gran cuestión que el texto de Roldán plantea es la relación entre filosofía de la historia y ética. En este caso, la autora enuncia la pregunta clave y sugiere algunas ideas que nos permiten introducir el debate: "Mi apuesta por la filosofía de la historia es una apuesta mediada por la ética. No propongo que la ética sustituya a la historia, pero sí que se sirva de ella para seguir buscando el rumbo que evite la catástrofe. De alguna manera, se trata de una simbiosis entre historia y ética ¿Podrá la ética dar un renovado tirón del pasado y hacer que el ángel de la historia al que aludía W. Benjamin vuelva la cara hacia el futuro sin dejarse arrastrar por el huracán del mal llamado progreso hacia su autodestrucción?"54. Para responder a esta pregunta me gustaría tomar prestadas unas palabras de Manuel Cruz: "La historia, pues, nos ayuda a vivir el presente de la única manera que le es dado hacerlo: ayudándonos a entenderlo [...] Por un conocimiento del presente, como es obvio, pasan nuestras programaciones de futuro. Como también pasa por él la noticia del pasado"55. La historia debe ayudarnos a entender nuestra actualidad para orientar la acción o, dicho de otro modo, la reflexión sobre la historia es condición sine qua non de una acción responsable.

¿Por qué situar el problema de la relación entre la filosofía de la historia y la ética en el presente? Porque, en mi opinión, tanto el fracaso de la filosofía especulativa como el de la filosofía crítica pueden explicarse, hasta cierto punto, por su incapacidad para medirse con la actualidad. Así, lo propio de la filosofía especulativa de la historia era colocar el acento en el futuro, entendido como el momento de realización de la Aufklärung, de la revolución proletaria o del estadio positivo. El presente era el instante previo, el espacio de posibilidad desde el que emergía el futuro: "Si nos preguntamos si vivimos en una época ilustrada, la respuesta es no, pero sí en una época de Ilustración. Todavía falta mucho para que los hombres, tal como están las cosas, considerados en su conjunto, puedan ser capaces o estén en situación de servirse bien y con seguridad de su propio entendimiento sin la guía de otro en materia de religión. Sin embargo, es ahora cuando se les ha abierto el espacio para trabajar libremente en este empeño"56. En el caso de la filosofía crítica, el énfasis en la explicación (que es común a autores tan diferentes como Ranke o Hempel) era correlativo a una definición del pasado como un objeto de conocimiento ajeno al presente desde el que el historiador escribe. Se suponía que existía un único pasado que podía ser descrito en términos objetivos. En definitiva, el acento se colocaba en el conocimiento del pasado en tanto que pasado, "objeto impávido a la espera de conocimiento"57.

Sin embargo, el pasado del historiador no es pretérito perfecto simple sino compuesto. El pasado sólo puede vivir a través de un presente que, además, es el lugar de la acción. Es por esta razón que es allí donde debemos plantear la pregunta por la ética. Para hacerlo, es necesario comenzar tomando algunas precauciones. Así, hay que evitar "el análisis del momento presente como si éste fuera precisamente en la historia el momento de la ruptura o el momento del balance, o del cumplimiento, o el de la aurora que retorna"58. El presente no es el momento de advenimiento de la historia, sino "un día como los otros, o más bien es un día que nunca es igual completamente a los demás"59. La filosofía de la historia debe, por tanto, colocarse al servicio de la actualidad no para legitimarla, sino para explicarla y para permitirnos modificarla. 
José Carlos Bermejo lo ha explicado con brillantez: "Será necesario partir de la situación presente, pero sin perder de vista, eso sí, que este presente nuestro no tiene sentido sin referencia al pasado, no ya porque el pasado pueda servir como fundamento del presente, sino porque precisamente lo que aquél nos pone de manifiesto es el carácter abierto del momento actual, su falta de fundamento, su permanente estado de indeterminación"60. Esta interpretación permite concebir la filosofía de la historia como una reflexión sobre el presente que se resume en la cuestión del "hoy" y en el problema del "nosotros": Que sommes-nous en ce temps qui est le nôtre? ${ }^{61}$.

¿En qué medida esta filosofía de la historia entendida como una ontología del presente puede ayudar a orientar la acción? Dicho de otro modo: ¿Qué función ética puede desempeñar una filosofía crítica? Como he señalado en las páginas anteriores, la filosofía debe ayudarnos a comprender la relación entre la actualidad y la historia que escribimos. Se trataría, como dice Manuel Cruz, de preguntar al que pregunta con el objetivo de asumir nuestra responsabilidad en la construcción del pasado: "Preguntar al que pregunta significa, por tanto, abrir estos interrogantes: ¿quiénes somos, en definitiva, los habitantes de ese presente? ¿Qué nos define? ¿A quiénes consideramos nuestros iguales en la historia?"62. Si tenemos en cuenta que nuestras inquietudes, pensamientos e ideas sobre el pasado nos dicen más a propósito de quiénes somos que de quienes éramos, entonces estaremos de acuerdo en que la filosofía de historia puede ser una herramienta fundamental para comprender la actualidad. Y esto es así porque, como ya he señalado, sólo un tipo de reflexión meta-histórica puede permitirnos comprender el sentido general de la historia que escribimos (más aún cuando nuestra reflexión sobre la historia adopta formas cada vez más variadas cuyo significado no puede ser aprehendido más que a través de algún tipo de reflexión global). Convertida en una forma de diagnóstico de nuestra actualidad, la filosofía de la historia podrá orientar la acción de la única manera que le es dado hacerlo: aportando el conocimiento necesario para que una acción responsable sea posible.

\section{Conclusión}

Con Entre Casandra y Clío sucede lo que con algunos relatos de Borges: en unas pocas páginas se encuentran resumidas las cuestiones que importan. ¿Podemos hablar de filosofía de la historia en el comienzo del siglo XXI? ¿Qué queda de la fe ilustrada en la posibilidad de conocer racionalmente el pasado? ¿Puede la reflexión sobre la historia fundamentar la acción? La respuesta a estas preguntas dependerá, en buena medida, de la concepción que cada uno tenga de la filosofía de la historia. Quienes consideran que el concepto define un tipo de reflexión meta-histórica que alcanzó su cenit con los sistemas de Hegel, Kant o Comte, probablemente entenderán que la Geschichtsphilosophie pertenece al pasado. Quienes consideramos que el concepto remite a una reflexión sobre y a partir de la historia entendemos, en cambio, que "todavía podemos hablar de filosofía de la historia"63. Teniendo en cuenta la crisis de las grandes narraciones tradicionales, el problema estriba en pensar qué sentido puede tener la reflexión filosófica sobre la historia en este momento que nos ha tocado vivir.

En este ensayo he propuesto algunas ideas al respecto. En mi opinión, la filosofía de la historia debe convertirse en una reflexión crítica orientada por la actualidad. En primer lugar, la filosofía 
debe ayudarnos a comprender el presente determinando qué tipo de reflexión histórica es la específica de nuestro hoy. ¿Por qué esa recurrente preocupación por la tradición y la memoria? ¿Qué es lo que hay detrás de nuestra inquietud por la identidad? ¿Por qué el creciente interés por objetos antes despreciados como la contingencia o el acontecimiento? La filosofía de la historia no debe limitarse a responder a cada una de estas cuestiones por separado, sino que tiene que decirnos algo sobre su significado. Tiene, por tanto, que ofrecernos una síntesis que nos permita comprender por qué pensamos la historia de una determinada manera y no de otra. Ese trabajo sobre la actualidad nos permitirá determinar lo que de específico tiene nuestra reflexión sobre la historia y, al mismo tiempo, nos ayudará a comprender quiénes somos. Así, conviene insistir en que la manera que nuestra sociedad tiene de pensar su historia dice mucho más a propósito de su presente que de su pasado. Por lo tanto, la reflexión sobre nuestro ser histórico debería, como he indicado en la última parte de este trabajo, ser condición imprescindible de una acción responsable.

En definitiva, estamos en un momento en el que urge construir una nueva filosofía de la historia. Quizá por ello, me gustaría concluir deseando que la autora de Entre Casandra y Clío, después de ofrecernos una brillante historia de la disciplina, se aplique al legado de Collingwood con el que cierra su libro: "construid vuestras historias y vuestras filosofías, aunque sepáis que no son definitivas"64.

\section{AGRADECIMIENTOS}

Quisiera agradecer a Manuel Cruz, Roberto R. Aramayo, José Carlos Bermejo y José Luis Moreno Pestaña la lectura atenta de mi texto. Un recuerdo especial merecen Jaime de Salas, cuyos interesantes comentarios a este ensayo me permitirán plantear nuevos problemas, y Concha Roldán, con quien pude discutir muchos de los temas aquí planteados. A todos ellos, muchas gracias.

\section{NOTAS}

1 Roldán, C. (2005): Entre Casandra y Clío: Una historia de la filosofía de la historia, Akal, Madrid, p.13.

2 Cruz, M. (1991): Filosofía de la historia. El debate sobre el historicismo y otros problemas mayores. Paidós, Barcelona.

${ }_{3}$ Bermejo, J. C. y Piedras, P. A. (1999): Genealogía de la Historia. Ensayos de Historia Teórica III. Akal, Madrid.

4 Suele distinguirse actualmente en alemán entre dos términos, Geschichtsphilosophie, para referirse a la filosofía de la historia en sentido estricto - como forma tradicional de entender esa disciplina durante una época - y Philosophie der Geschichte en un sentido más amplio que incluiría y partiría de las críticas de la denominada posthistoria; esto es, la última sería una manera no exenta de paradoja de volver a reflexionar sobre la historia "después del fin de la filosofía de la historia". Cfr. al respecto Rohbeck, J. Geschichtsphilosophie zur Einführung, Junius, Hamburg, 2004, p.19, citado por Roldán (2005), p. 220.

5 "La tarea de la filosofía es concebir lo que es, pues lo que es es la razón. En lo que respecta al individuo, cada uno es, de todos modos, hijo de $s u$ tiempo; de la misma manera, la filosofía es su tiempo aprehendido en pensamientos. Es igualmente insensato creer que una filosofía puede ir más allá de su tiempo presente como que un individuo puede saltar por encima de su tiempo, más allá de Rodas": Hegel, G. W. F. (1820): Principios de la filosofía del derecho o derecho natural y ciencia política, Edhasa, Barcelona, 1999, p. 61.

6 Roldán, C. (2005), p. 17. 
7 Roldán, C. (2005), 16.

8 Concepción que, siguiendo a Manuel Cruz, estaría tentado de llamar filosofía idealista de la historia: "Nada impide, por tanto, aceptar la crisis o el debilitamiento de las grandes narraciones de teología y filosofía de la historia heredadas de siglos pasados [...] Su error habría sido empeñarse en buscar un fundamento objetivo, exterior, a la esperanza o a la ilusión: al sentido positivo de desarrollo, en definitiva. A la primera acometida de los hechos mismos, todas ellas habrían mostrado su condición de ideales: ideal de la revolución proletaria, ideal del desarrollo económico indefinido, ideal de la democracia burguesa..., incapaces de sobrevivir a la refutación", en: Cruz, M. (1991), pp. 30-31.

${ }^{9}$ Walsh, W. H. (1978): Introducción a la filosofía de la historia, Siglo XXI, Madrid, p. 12; y Danto, A. C. (1989): Historia y narración. Ensayos de filosofía analítica de la historia, Paidós, Barcelona, p. 29. A esta disquisición terminológica, apuntada en pp. 13-14, dedica Roldán (2005) las pp. 117-122.

10 Ya en 1944, Ernst Cassirer señalaba el fracaso de la filosofía especulativa de la historia: "Espero que a partir de estas observaciones podréis juzgar si podemos continuar hablando de una filosofía de la historia. No podemos comprender este término en su sentido metafísico anterior [...] No podemos descubrir mediante métodos simplemente especulativos los hechos o las leyes de la historia", en: Cassirer, E. (1944): "La Philosophie de l'Histoire", en: L'idée de l'histoire. Les inédits de Yale et autres écrits d'exil, Cerf, Paris, 1988, p. 52.

11 Roldán, C. (2005): p. 17.

12 Aron, R. 1969. La philosophie critique de l'histoire, Vrin, Paris, p. 15.

13 Cfr. Roldán. C. (2005), pp. 122-128.

14 Bourdeau, L. 1888. L'histoire et les historiens: essai critique sur l'histoire considérée comme science positive, Alcan, Paris.

15 Cfr. Roldán. C. (2005), pp. 128-135.

16 Roldán, C. (2005), p.18.

17 Roldán, C. (2005), pp. 16-17.

18 Cassirer, E. (1944), p. 52. A. Gómez Ramos parte de está contradicción en su libro Reivindicación del centauro. Actualidad de la filosofía de la historia, Akal, Madrid, 2003; citado por Roldán (2005) p. 220.

19 Roldán, C. (2005), p.49.

20 Como señala Danto (1989, p. 29): "Un filósofo de la historia trata de proporcionar una explicación del conjunto de la historia".

21 Según Spengler, la idea de una historia universal es una creación occidental: "La historia universal es nuestra imagen del mundo, no la imagen de la "humanidad". El indio y el antiguo no se representaban el mundo en su devenir. Y cuando se extinga la civilización del Occidente, acaso no vuelva a existir otra cultura y, por lo tanto, otro tipo humano para quien "la historia universal" sea una forma tan enérgica de la conciencia vigilante", en: Spengler, O. (1918): La decadencia de Occidente, Espasa- Calpe, Madrid, 1966, p. 41.

22 Ortega y Gasset, J. (1966): "Proemio", en: Spengler, O. (1918), p. 13.

23 "En este libro se acomete por vez primera el intento de predecir la historia", Spengler, O. (1918, p. 25)

24 En Ibíd.

25 En Ibíd., p. 48.

26 En Ibíd., p. 71.

27 En Ibíd., p. 48.

28 Walsh, W. H. (1978), p. 12

${ }^{29}$ Roldán, C. (2005), p.116.

30 Cassirer, E. (1944), p. 52.

31 Muguerza, J. en "Prólogo" a: C. Roldán (2005), p. 5 .

32 Cruz, M. (1991), pp. 24-25.

33 Roldán, C. (2005), p. 17.

34 Citado en: Roldán, C. (2005), p.18.

35 Cruz, M. (1991), p. 16.

36 Cfr. Roldán, C. (2005), p. 207.

${ }^{37}$ Iggers, G. G. (1995): La ciencia histórica en el siglo XX. Las tendencias actuales. Una visión panorámica y crítica del debate internacional, Idea Books, Barcelona, p. 11.

38 Monod, G. (1876): "Du progrès des études historiques en France”, en : G. Bourdé y H. Martin (1983): Les écoles historiques, Seuil, Paris, pp. 168 y 170 .

39 Hartog, F., Revel, J. (2001) : Les usages politiques du passé, Éditions de l'EHESS, Paris, p. 14.

40 Guizot, F. (1820): Du gouvernement de la France depuis la Restauration et du ministère actuel, Librairie Française de Ladvocat, Paris, p. 210.

41 Michelet, J. (1869): “ Préface de 1868 à l'Histoire de la Révolution Française”, en: Histoire de la Révolution Française, Gallimard, Paris, 1952, p. 11.

42 Thierry, A. (1835): Dix ans d'études historiques, Just Tessier, Paris, p. XVI.

43 Bourdieu, P. (2000): Propos sur le champ politique, Presses Universitaires de Lyon, Lyon, p. 52.

44 De acuerdo con Iggers, dicho equilibrio estuvo basado en la supremacía de la "orientación científica". Según este autor, el pensamiento histórico ha tomado dos grandes orientaciones durante el siglo XX. (a) una orientación científica (representada por la Social History, la escuela de Annales o el marxismo) que propuso una crítica del historicismo con el objetivo de reforzar la posibilidad de una historia científica; y (b) una corriente filosófica que, desde finales del siglo 
XIX, ha planteado una crítica radical a la historia científica. Esta segunda corriente sólo adquirió un protagonismo importante a finales del siglo $\mathrm{XX}$ : "Sin embargo, ha sido solo ha finales del siglo XX cuando las dudas provocadas por este desafío han afectado seriamente al trabajo de los historiadores", en: Iggers, G. C. (1997): Historiography in the Twentieth Century. From Scientific Objectivity to the Postmodern Challenge, Wesleyan University Press, Connecticut, p. 5.

45 Koselleck, R. (2002): The practice of conceptual history: timing history, spacing concepts, Stanford University Press, Stanford, p. 14.

46 White, H. (1973): Metahistory. The Historical Imagination in Nineteenth- Century Europe, The Johns Hopkins University Press, Baltimore \& London (hay trad. cast.: Metahistoria. La imaginación histórica en la Europa del siglo XIX, México, Fondo de Cultura Económica, 1992).

47 Stone, L. (1979): "The Revival of Narrative: Reflections on a New Old History", History and Theory, 85, pp. 3-24.

48 Rorty, R. (ed., 1967): The Linguistic Turn. Recent Essays in Philosophical Method, The University of Chicago Press, Chicago.

49 White, H. (1974): “The Historical Text as Literary Artifact”, en: H. White (1978): Tropics of discourse. Essays in cultural criticism. The Johns Hopkins University Press, Baltimore \& London, p. 82 (hay trad. cast. de Verónica tozzi y Nicolás Lavagnino, El texto histórico como artefacto literario, Paidós, Barcelona, 2003).

50 "Los límites de mi lenguaje son los límites de mi mundo", en: Wittgenstein, L. (1927): Tractatus Logico- Philosophicus (trad. Pierre Klossowski), Gallimard (coll. Idées), Paris, p. 141.

51 Foucault, M. (1975): Vigilar y castigar. Nacimiento de la prisión, Siglo XXI, Madrid, 1992 , p. 34.

52 Breisach, E. (2003): On the Future of History: the postmodernist challenge and its aftermath, Chicago University Press, Chicago.
53 En este sentido, estoy de acuerdo con Bourdieu cuando escribe: "No hay contradicción, pese a las apariencias, en luchar al mismo tiempo contra la hipocresía mistificadora del universalismo abstracto y a favor del acceso universal a las condiciones de acceso a lo universal, objetivo primordial de todo verdadero humanismo que la predicación universalista y la (falsa) subversión nihilista tiene en común olvidar", en: Bourdieu, P. 1997. Meditaciones pascalianas, Anagrama, Barcelona, p. 98.

54 Roldán, C. (2005), p. 17-18.

55 Cruz, M. (1991), p. 44.

56 Kant, I. (1784): "Ideas para una historia universal en clave cosmopolita", en: Ideas para una historia universal en clave cosmopolita y otros escritos sobre filosofía de la Historia, ed. de R.R. Aramayo en cotraducción con C. Roldán, Tecnos, Madrid, 1987, p. 15.

57 Cruz, M. (1991), p. 13.

58 Foucault, M. (1983): "Estructuralismo y postestructuralismo", en: Estética, ética y hermenéutica. Obras esenciales. Volumen III, Paidós, Barcelona, 1999, p. 325.

${ }^{59}$ Foucault (1983), p. 325

60 Bermejo, J. C.; Piedras, P. A. 1999. pp. 5-6.

61 Foucault, M. 1988. "La technologie politique des individus", en: Michel Foucault. Dits et écrits II, 1976-1988, Gallimard, Paris, 2001, pp. 1632.

62 Cruz, M. op. cit, p. 26- 27.

63 Roldán, C. (2005), p. 17.

64 Roldán, C. (2005), p. 207. En este sentido es obligado citar el trabajo de Roldán recientemente publicado en un volumen colectivo dedicado a Javier Muguerza que lleva por título Disenso e incertidumbre; ver C. Roldán, "Nuevas vueltas a la historia. Por una filosofía de la historia doblada de ética", en J. F. Álvarez y R.R. Aramayo, Disenso e incertidumbre. Un homenaje a Javier Muguerza, Plaza y Valdés, Madrid, 2006, pp. 519-546. 